\title{
On ideals consisting of joint topological divisors of zero
}

by

ZBIGNIEW SEODKOWSKI (WarsZaWa)

Abstract. Let $A$ be a commutative Banach algebra with unit e. Let $l(A)$ designate the collection of all closed ideals of $A$ consisting of joint topological divisors of zero. The main result of this paper states that any ideal $I_{\epsilon} t(A)$ is contained in a maximal ideal of $A$, which also belongs to $l(A)$. This solves a problem posed in [3].

All algebras considered in this paper are commutative complex unitary algebras. The unit element of such an algebra will be denoted by $e$. Let $A$ be such a Banach algebra. A non-void subset $S \subset A$ is said to consist of joint topological divisors of zero if there is a net $\left(b_{\alpha}\right) \subset A$, $\left\|b_{a}\right\|=1$ such that $\lim b_{a} a=0$ for each $a \in S$. So a set $S$ consists of joint topological divisors of zero if and only if for each finite subset $\left(a_{1}, \ldots, a_{n}\right)$ $\subset \$$ we have

$$
\delta\left(a_{1}, \ldots, a_{n}\right)=0
$$

where

$$
\delta\left(a_{1}, \ldots, a_{n}\right)=\inf _{\|b\|=1} \sum_{i=1}^{n}\left\|b a_{i}\right\| .
$$

A closed ideal $I \subset A$ is said to be an $t$-ideal if it consists of joint topological divisors of zero. The family of all $l$-ideals of $A$ is denoted by $\imath(\mathcal{A})$.

Let $\mathscr{L}(A)=\ell(A) \cap \mathfrak{M}(A)$, where $\mathfrak{M}(A)$ is the maximal ideals space of $A$. The above concept has been introduced in [3], where it was shown that $\mathcal{L}(A)$ is a closed subset of $\mathfrak{M}(A)$ containing the Shilov boundary. It was shown also that every $t$-ideal $I$ is contained in a maximal $t$-ideal $J$, i.e. an ideal $J \in \mathcal{Z}(A)$ such that if $J_{1}$ is an ideal in $l(A)$ and $J \subset J_{1}$ then $J_{1}=J$. It was not known, however, and it was asked in [3] whether every t-maximal ideal is a maximal ideal in $A$, i.e. whether such an ideal belongs to $\mathcal{E}(A)$.

In this paper we give an affirmative answer to this question. As a corollary we prove also that if a Banach algebra $B$ is an extension of $A$, 
i.e. if $B$ contains isomorphically $A$ (as a closed subalgebra, with topology induced on $A$ by $B$ equivalent to the original topology on $A$ ) and the unit of $A$ serves as the unit element of $B$, then any multiplicative and linear functional $f \in \mathscr{L}(A)$ extends to a member $\bar{f} \in \mathscr{L}(B)$ (we identify here the multiplicative linear functionals with their kernels). This gives an answer to another question posed in [3].

The author is indebted to Professor W. Zelazko for calling his attention to these problems and for formulating the basic Lemma 3.

Limara 1. Let $I$ be an ideal of an algebra $R$, and $p$ a submultiplioative seminorm on $R$. Then

(a) for any $r$ in $R$ the set

$$
M(r)=\{C \geqslant 0: p(r i) \leqslant C p(i) \text { for each } i \in I\}
$$

is non-void, and the function on $R$

$$
q(r)=\inf M(r)
$$

is a submultiplicative seminorm on $R$.

(b) If, in addition, there is an element $y$ in $I$ such that $p(y) \neq 0$, then $q(e)=1$.

Proof. Since the seminorm $p$ is submultiplicative, the number $p(r)$ belongs to the $M(r)$ and so it is a non-void set. We notice that the set $M(r)$ is closed, and hence $q(r)$ belongs to $M(r)$. This means that

$$
p(r i) \leqslant q(r) p(i) \text { for all } r \text { in } R \text { and } i \text { in } I .
$$

For $r_{1}, r_{2}$ in $R$ and $i$ in $I, r_{2} i$ belongs to $I$ and from (5) we have

$$
p\left(\left(r_{1} r_{2}\right) i\right)=p\left(r_{1}\left(r_{2} i\right)\right) \leqslant q\left(r_{1}\right) p\left(r_{2} i\right) \leqslant q\left(r_{1}\right) q\left(r_{2}\right) p(i) .
$$

This implies $q\left(r_{1} r_{2}\right) \leqslant q\left(r_{1}\right) q\left(r_{2}\right)$. Similarly

$$
p\left(\left(r_{1}+r_{2}\right) i\right) \leqslant p\left(r_{1} i\right)+p\left(r_{2} i\right) \leqslant\left(q\left(r_{1}\right)+q\left(r_{2}\right)\right) p(i)
$$

and so

$$
q\left(r_{1}\right)+q\left(r_{2}\right) \geqslant q\left(r_{1}+r_{2}\right) .
$$

Since $q$ is obviously a homogeneous function, it is a submultiplicative seminorm.

The simple proof of (b) can be omitted.

LEMNA 2. Let $R$ be an algebra, $q$-a submultiplicative seminorm on $R$, and $x$ - an element of $R$ such that for any complex $\lambda$ there exists a positive number $\varepsilon_{\lambda}$ satisfying.

$$
q(r(\lambda e-x)) \geqslant q(r)
$$

for every $r$ in $R$. Then $q(e)=0$.
Proof. Suppose that $q(e) \neq 0$. We define a Banach algebra $A$ by completing the normed algebra $R /$ ker $q$, with the norm induced by $q$. We denote this norm by \|\| . There is a natural map $\varphi: R \rightarrow A$, namely

$$
\varphi(r)=r+\operatorname{ker} q .
$$

We put $\varphi(x)=a$. It easily follows from (6) that for each complex $\lambda$ and for each $b$ in $A$. We have

$$
\|b(\lambda e-a)\| \geqslant\|b\| \varepsilon_{\lambda} .
$$

This means that for every complex $\lambda$ the element $\lambda e-a$ is not a topological divisor of zero in $A$. But this is impossible for, say $\lambda$ belonging to the boundary of the spectrum of $a$ in $A$ (cf. [2]) and the lemma follows.

LEMvia 3. Let $A$ be a Banach algebra, $a_{1}, \ldots, a_{n}, a \in A$, and suppose that subset $\left\{a_{1}, \ldots, a_{n}\right\}$ consists of joint topologioal divisors of zero. Then there is a complex number $\lambda$ such that the subset $\left\{a_{1}, \ldots, a_{n}, \lambda e-a\right\}$ consists of joint topologioal divisors of zero.

Proof. Suppose that the lemma is false. Then there is a Banach algebra $A$ and elements $a_{1}, \ldots, a_{n}, a$ in $A$, such that for each integer $k$ there is a $b_{l}$ in $A$, and for any complex $\lambda$ there is a positive $\varepsilon_{\lambda}$ satisfying following relations:

$$
\begin{gathered}
\left\|a_{1}\right\|, \ldots,\left\|a_{n}\right\|,\|a\| \leqslant 1, \\
\left\|b_{k}\right\|=1 \quad \quad k=1,2, \ldots, \\
+\left\|a_{n} d\right\|+\|(\lambda e-a) d\| \geqslant \varepsilon_{\lambda}\|d\| \quad \text { for } a \\
\lim _{k}\left\|b_{l_{t}} a_{i}\right\|=0, \quad i=1,2, \ldots, n .
\end{gathered}
$$$$
\left\|a_{1} d\right\|+\ldots+\left\|a_{n} d\right\|+\|(\lambda e-a) d\| \geqslant \varepsilon_{\lambda}\|d\| \quad \text { for and } d \text { in } A
$$

We denote by $R$ the algebra $O\left[x_{1}, \ldots, x_{n}, x, y\right]$ of all polynomials in $x_{1}, x_{2}, \ldots, x_{n}, x, y$

$$
r=\sum_{i_{1}, \ldots, i_{n}, i, j \geqslant 0} \alpha_{i_{1}, \ldots, i_{n}, i, j} x_{1}^{i_{1}} \ldots x_{n}^{i_{n}} \infty^{i} y^{j}
$$

with complex coefficients $\alpha_{i, \ldots, i, i, j}$ (the summation is finite). For each integer $k$ we define a mapping of $R$ into $A$ given by

$$
r \rightarrow h_{k}(r)=\sum_{1_{1}, \ldots, i_{n}, i_{1}, \geqslant 0} \alpha_{i_{1}, \ldots, i_{n}, i, j} a_{1}^{i_{1}} \ldots a_{n}^{i_{n}} a^{i} b_{k}^{j} .
$$

and a mapping $N_{\gamma_{0}}$ of $R$ into non-negative real numbers, given by

$$
N_{k}(r)=\left\|h_{k}(r)\right\| \text {. }
$$

It is easy to see that the mapping (12) is an algebraic homomorphism and in consequence; formula (13) gives a submultiplicative seminorm on $R$. 
If $d=h_{k}(y r)$ where $r$ belongs to $R$ and $k=1,2, \ldots$, then

$\left\|a_{i} d\right\|=\left\|h_{k}\left(x_{i}\right) h_{k}(y r)\right\|=\left\|h_{k}\left(x_{i} y r\right)\right\|=N_{k}\left(x_{i} y r\right), \quad i=1,2, \ldots, n$,

$\|d\|=N_{k}(y r)$,

$\|(\lambda e-a) d\|=\left\|h_{k}(\lambda e-x) h_{k}(y r)\right\|=N_{k}((\lambda e-x) y r)$

and from (9) follows

$$
N_{k}\left(x_{1} y r\right)+\ldots+N_{k}\left(x_{n} y r\right)+N_{k}((\lambda e-\infty) y r) \geqslant \varepsilon_{\lambda} N_{k}(y r) .
$$

We notice that $N_{k}(r) \leqslant o_{r}, r \in R, k=1,2, \ldots$, where

$$
o_{r}=\sum_{i_{1}, \ldots, i_{n}, i, j \geqslant 0}\left|a_{i_{1}, \ldots, i_{n}, i, j}\right|
$$

for any $r$ in $R$ of the form (11). Hence any mapping $N_{k}$ belongs to the Cartesian product of the collection $\left\{\left[0, c_{r}\right]: r \in R\right\}$, considered as a set of mappings. This product is compact relative to the product topology; hence we can choose a subnet $\left\{p_{m}: m \in M\right\}$ of the sequence $\left\{N_{k}: k\right.$ $=1,2, \ldots\}$ which converges to a point $p$ of the product. (This subnet need not be a sequence; $(M, \leqslant)$ is a directed set.)

We recall that a net in a product space converges to a point if and only if its projection in each coordinate space converges to the projection of the point ([1], Chap. 3., Theorem 4), hence for any $r$ in $R$

$$
\lim _{m} p_{m}(r)=p(r) \text {. }
$$

Any $p_{m}$ is equal to some $N_{k}$, and therefore it is a submultiplicative seminorm. Each subnet of a converging net converges to the limit of the net ([1], p. 74, (a)). For any $r$ in $R$ the net $\left\{p_{m}(r): m \in M\right\}$ is a subnet of the sequence $\left\{N_{k}(r): k=1,2, \ldots\right\}$ and from (10), (8)

$$
\lim _{k} N_{k}\left(r y x_{i}\right)=\lim _{k}\left\|h_{k}\left(r y x_{i}\right)\right\|=\lim _{k}\left\|b_{k} a_{i} h_{k}(r)\right\|
$$

$$
\leqslant\left(\lim _{k}\left\|b_{k} a_{i}\right\|\right) c_{r}=0, \quad i=1,2, \ldots, n
$$

and

hence

$$
\lim _{k} N_{k}(y)=\lim _{k}\left\|b_{k}\right\|=1
$$

Any $p_{m}$ is equal to some $N_{k}$, and hence by (14) we get

$$
p((\lambda e-x) y r) \geqslant \varepsilon_{\lambda} p(y r) \text {. }
$$

Observe that the ideal $I=y R$, the seminorm $p$ and, by (16), the member $y$ of $I$ satisfy the conditions of Lemma 1 ; hence the function $q(r)$ given by formula (4) is a submultiplicative seminorm on $R$ and, by (16) and condition (b) of Lemma 1 ,

$$
q(e)=1
$$

We can write

and therefore

$$
q(r) p\left(r_{1} y\right) \geqslant p\left(r r_{1} y\right)
$$

By (17)

$$
q((\lambda e-x) r) p\left(r_{1} y\right) \geqslant p\left((\lambda e-x) r r_{1} y\right)
$$

$$
p\left((\lambda e-\infty) r r_{1} y\right) \geqslant \varepsilon_{\lambda} p\left(r r_{1} y\right)
$$

and by the definition of $q$ (formulas (3) and (4)) we get

$$
q((\lambda e-x) r) / \varepsilon_{\lambda} \geqslant q(r) \text {. }
$$

Now by Lemma $2 q(e)=0$ but by (16) this is impossible, which gives a contradiction completing the proof.

LEMMA 4. Let $A$ be a Banach algebra, let $F=\left\{a_{1}, \ldots, a_{n}\right\}$ be a finite subset of $A$ which consists of joint topological divisors of zero, and let a belong to $A$. Then the set

$$
Z=Z(F, a)=\left\{\lambda \epsilon C: \delta\left(a_{1}, \ldots, a_{n}, e-a\right)=0\right\}
$$

is a non-void compact subset of the complex plane.

Proof. $Z$ is non-void from Lemma 3 .

It is easy to see that no invertible element of $A$ is a topological divisor of zero; if a finite subset of $A$ consists of joint topological divisors of zero, then each of its elements is a topological divisor of zero. These remarks imply that $Z$ is contained in the spectrum of $a$, and hence $Z$ is bounded.

Now it is sufficient to prove that $Z$ is a closed subset of the complex plane. Let $\lambda$ be an accumulation point of $Z$; then for a given $\varepsilon>0$ there is a $\lambda_{1}$ in $Z$ such that $\left|\lambda-\lambda_{1}\right|<\varepsilon / 2$. The subset $\left\{a_{1}, \ldots, a_{n}, \lambda_{1} e-a\right\}$ consists of joint topological divisors of zero. Hence there is a $d$ in $A$ with $\|d\|=1$ such that

$$
\left\|a_{1} d\right\|+\ldots+\left\|a_{n} d\right\|+\left\|\left(\lambda_{1} e-a\right) d\right\| \leqslant \varepsilon / 2
$$

and we have

$$
\begin{aligned}
\left\|a_{1} d\right\|+\ldots+ & \left\|a_{n} d\right\|+\|(\lambda e-a) d\| \leqslant\left\|a_{1} d\right\|+\ldots+\left\|a_{n} d\right\|+ \\
& +\left\|\left(\lambda_{1} e-a\right) d\right\|+\|(\lambda e-\lambda e) d\| \leqslant \varepsilon / 2+\left|\lambda-\lambda_{1}\right| \cdot\|d\|<\varepsilon .
\end{aligned}
$$

This means that $\delta\left(a_{1}, \ldots, a_{n}, \lambda \theta-a\right) \leqslant \varepsilon$ for every $\varepsilon>0$, and so $\lambda$ belongs to $Z$.

THEOREm. Let $A$ be a commutative complex Banach algebra with unit e. Let $I \in Z(A)$. Then there is a $J \in \mathcal{E}(A)$ such that $I \subset J$.

Proof. Let $I$ be an $t$-ideal of codimension greater than one. This means that there is an $a$ in $A$ such that for any complex $\lambda$ the element 
$\lambda e-a$ does not belong to $I$. Since, by Lemma 3 of [3] any $t$-ideal is contained in an $t$-maximal ideal, it is sufficient to prove that there is an $l$-ideal $J$ which properly contains $X$.

Let $F_{1}, F_{2}$ be finite subsets of $I$ and $F_{1} \supset F_{2}$. It is easy to see that

$$
Z\left(F_{1}, a\right) \subset Z\left(F_{2}, a\right)
$$

where the set $Z(F, a)$ is given by formula (19). Hence for any finite family $\left\{F_{1}, \ldots, F_{n}\right\}$ of finite subsets of $I$

$$
Z\left(F_{1}, a\right) \cap \ldots \cap Z\left(F_{n}, a\right)>Z\left(F_{1} \cup \ldots \cup F_{n}, a\right) .
$$

This means that the family of all subsets $Z\left(F^{\prime}, a\right)$, where $I^{\prime}$ is any finite subset of $I$ has the finite intersection property. Any $I F$ in $I$ consists of joint topological divisors of zero. Hence by Lemma 4 any $Z(F, a)$ is a non-roid compact set. Thus the family has a non-void intersection. Let $\lambda_{0}$ belong to the intersection. We notice, that the set $I \cup\left\{\lambda_{0} e-a\right\}$ consists of joint topological divisors of zero. By Lemmas 1 and 2 in [3], which jointly state that any subset of $A$ consisting of joint topological divisors of zero is contained in an $t$-ideal, there is an $t$-ideal $J$ which contains $I$ and $\lambda_{0} e-a$. The inclusion is proper, because $\lambda_{0} e-a$ does not belong to $I$. So we have obtained a contradiction of the assumption that $I$ is a $l$ maximal ideal.

Remark. Since every maximal ideal is a prime ideal, Proposition 2 in [3], which states that every $t$-maximal ideal is a prime ideal, follows immediately from the Theorem.

CoROLLARY. If $f$ is a functional in $\mathscr{L}(A)$ and $B$ is an extension of $A$, then $f$ extends to a member $F$ of $\mathcal{E}(B)$.

Proof. Since any $l$-ideal in $A$ is contained in an $l$-ideal of $B$ (Proposition 1 of [3]), the kernel of $f$ is contained in an $t$-ideal of $B$. By the Theorem, the ideal is contained in an ideal in $\mathscr{E}(B)$. The multiplicative-linear functional $F$ in $\mathfrak{M}(B)$ coresponding to this ideal extends $f$ and belongs to $\mathscr{e}(B)$.

\section{References}

[1] J. L. Kelley, General topology, 1964.

[2] I. M. Gelfand, D. A. Raikov, G. E. Shilov, Oommutative normed rings, Now York 1964.

[3] W. ̇̇elazko, On a certain class of non-removable ideals in Banach algebras, Studia Math. 44 (1972), p. 87-92.

WARSAW UNIVHRSITY,

DEPARTMENT OF MATHEMATICS

\section{Separability of orbits \\ of functions on locally compact groups* \\ by \\ F. PORTA. (Urbana, Ill.), L. A. RUBEL (Urbana, Ill.) and A. T. SHITLDS (Ann Arbor, Mich.)}

Abstract. Let $G$ bo a locally compact group. First, if $f \in L^{\infty}(G)$ has a separable orbit under loft translation by olements of $G$, then $f$ is locally a.o. equal to a bounded and uniformly continuous function on $G$. Secondly, if $f \in L_{\text {loo }}^{\infty}(G)$ has a separablo orbit, then $f$ is locally a.e. equal to a continuous function on $G$.

1. Notation. Let $G$ be a locally compact group, $d x$ a left invariant Haar measure $\left(\int_{G} f(s x) d x=\int_{G} f(x) d x\right)$. We do not assume that $G$ is compact or Abelian or separable. For $s \in G$ and $f$ a function on $G$, the left translate of $f$ by $s$ is the function $(\gamma(s) f)(x)=f\left(s^{-1} x\right)$. We also write $f_{s}=\gamma(s) f$. For $\mu$ a measure on $G$, define $(\mu * f)(x)=\int_{\sigma} f\left(s^{-1} x\right) d \mu(s)$ whenever this makes sense. In particular, for two complex-valued functions $f$ and $g$ on $G$ we have $(g * f)(x)=\int_{\theta} f\left(s^{-1} x\right) g(s) d s$, which exists when $f\left(s^{-1} \infty\right) g(s) \epsilon L^{1}$. Also, if $\varepsilon_{u}$ denotes the unit mass at $u \in G$, then $\left(\varepsilon_{u} * f\right)(x)$ $=(\gamma(u) f)(x)$; i.e. $\varepsilon_{u} * f=\gamma(u) f$. Olearly, then, $\gamma(u)(f * g)=(\gamma(u) f) * g$ whenever both members malie sense.

The space $L^{\infty}(G)$ is the usual space of classes of bounded measurable functions. The space $L_{\text {loo }}^{\infty}(G)$ is the space of classes of measurable functions on $G$ that are bounded on compact subsets of $G$. Two functions belong to the same equivalence class if they agree except at most on a set that intersects every compact set in a set of zero measure. The topology of $I_{100}^{\infty}(G)$ is given by the seminorms

$$
\|f\|_{K}=\operatorname{ess} \sup \{|f(\infty)|: \infty \epsilon K\}
$$

as $K$ runs over the compact subsets of $Q$. Wo use the phrase "locally a.e." to mean "almost everywhere on each compact subset of $G$ ". By $O_{B O}(G)$ we mean the class of functions that are bounded and uniformly continuous on $G$.

2. Statements of results. Our first result is related to known results, but does not appear to be explicity stated in the literature. It was established independently for the circle group by T. Kaczynski (unpubli-

* The research of the authors was partially supported by different grants from the National Soience Foundation. 